\title{
Análisis del comportamiento acústico y optimización del material del escape de automóviles livianos equipados con turbo compresor mediante herramientas de dinámica de fluidos computacionales.
}

Analysis of the acoustic behavior and optimization of the material of the automobile exhaust lighter equipped with turbo compressor using tools from computational fluid dynamics.

Paúl Montúfar Paz. ${ }^{1}$, Rodrigo Rigoberto Moreno Pallares. ${ }^{2}$, Luis Santiago Choto Chariguaman. ${ }^{3}$, Luis Fernando Buenaño Moyano. ${ }^{4}$ \& Miguel Angel Escobar Guachambala. ${ }^{5}$

Recibido: 08-03-2017 / Revisado: 08-05-2017 Aceptado: 13-06-2018/ Publicado: 01-07-2018

\begin{abstract}
.
DOI: https://doi.org/10.33262/cienciadigital.v2i3.175

In the present research project was developed in a pre-silencer and muffler of the Zotye T600 vehicle assembled by CIAUTO in Ambato city, an analysis of computational fluids dynamics and acoustics through computer-aided engineering with the software ANSYS CFX and ANSYS ACT respectively. First, the geometry was generated by means of drawing and 3D scanning with the help of the SpaiceClaim CAD software. The input parameters for the simulations such as speed, temperature and speed of the motor correspond to values collected by means of suitable measuring instruments, in versions $1.5 \mathrm{~T}$ and 2.0T of the vehicle. Secondly, the CFD simulation was performed in ANSYS CFX since it is the most complete ANSYS solver in heat transfer in fluid-solid interface to analyze, according to the results obtained in the silencer, several design alternatives were proposed, which were evaluated in their operation and selected 2 of them, compared with the original by means of CFD and the graph of transmission loss, thus determining the advantages and disadvantages of the alternatives. It was observed in the acoustic simulation with respect to the transmission loss graph that there is no significant difference between the original design and the alternatives, as a result of this

${ }^{1}$ Escuela Superior Politécnica de Chimborazo, Chimborazo, Ecuador, paul.montufar@espoch.edu.ec.

${ }^{2}$ Escuela Superior Politécnica de Chimborazo, Chimborazo, Ecuador, rodrigo.moreno@espoch.edu.ec.

${ }^{3}$ Escuela Superior Politécnica de Chimborazo, Chimborazo, Ecuador, luis.choto@espoch.edu.ec.

${ }^{4}$ Escuela Superior Politécnica de Chimborazo, Chimborazo, Ecuador, lfbuenanio@espoch.edu.ec.

${ }^{5}$ Escuela Superior Politécnica de Chimborazo, Chimborazo, Ecuador, maescobar@espoch.edu.ec.
\end{abstract}


it was concluded that the alternatives produce a benefit without a significant increase in the noise produced by the engine combustion. It should be noted that the generation and evaluation of alternatives using CAE software do not replace the experimental tests which must be carried out, however it helps to simplify the prototyping process.

Keywords: Words: Silencer, Acoustics, Finite Element, Exhaust System, CFD.

\section{Resumen.}

En el presente proyecto de investigación se desarrolló en un pre-silenciador y silenciador del vehículo Zotye T600 ensamblado por CIAUTO en la ciudad de Ambato, un análisis de mecánica computacional de fluidos y acústico mediante ingeniería asistida por ordenador con el software ANSYS CFX y ANSYS ACT respectivamente. En primer lugar, se generó la geometría por medio de levantamiento de planos y escaneo 3D con la ayuda del software CAD SpaiceClaim. Los parámetros de entrada para las simulaciones tales como velocidad, temperatura y velocidad de giro del motor corresponden a valores recolectados mediante instrumentos de medición adecuados, en las versiones 1,5T y 2,0T del vehículo. En segundo lugar, se realizó la simulación CFD en ANSYS CFX puesto que es el solver de ANSYS más completo en transferencia de calor en interfaz fluido-sólido para analizar, de acuerdo a los resultados obtenidos en el silenciador se plantearon varias alternativas de diseño las mismas que fueron evaluadas en su funcionamiento y seleccionadas 2 de ellas, comparadas con el original por medio de CFD y la gráfica de pérdida de transmisión, determinando de esta manera las ventajas y desventajas de las alternativas. Se observó en la simulación acústica con respecto a la gráfica de pérdida de transmisión que no existe una diferencia significativa entre el diseño original y las alternativas, como consecuencia de esto se concluyó que las alternativas producen un beneficio sin un aumento significativo del ruido producto de la combustión del motor. Cabe recalcar que la generación y evaluación de alternativas mediante software CAE no reemplazan las pruebas experimentales las cuales deben realizarse, sin embargo, ayuda a la simplificación del proceso de prototipado.

Palabras Claves: Silenciador, Acústica, Elemento Finito, Sistema de Escape, CFD

\section{Introducción.}

La reducción de peso del vehículo puede ser lograda usando materiales más ligeros o reduciendo el tamaño de los componentes existentes. Los investigadores de "VE Commercial Vehicles Ltd." En India utilizaron este enfoque para diseñar un sistema de escape en un vehículo comercial utilizando la herramienta Fluent de ANSYS CFX, Resultando en una reducción del $14.1 \%$ del tamaño y volumen, así como una reducción del peso del 2\%. (SAE, 2014) 
La optimización de los silenciadores es uno de los retos más críticos de los ingenieros automotrices. Convencionalmente, el diseño de los silenciadores incluye los desafíos físicos más exhaustivos para varias combinaciones de silenciadores variando el diámetro de la tubería/ tamaño de las perforaciones/ posición del deflector/ volumen de amortiguación, etc. (SAE, 2014) Este proceso puede ser simplificado con la utilización de herramientas de simulación.

El uso de las herramientas de simulaciones ha mejorado el proceso de balancear el conflicto de los requerimientos tales como la contrapresión en el sistema de escape y el ruido. Valores altos de contrapresión indican una gran resistencia al flujo de los gases de escape. (Paucar Quinteros \& Toapanta Jaramillo, 2011) consideraciones teóricas

\section{Ruido.}

Junto a las emisiones contaminantes y el consumo, el ruido es considerado uno de los problemas más relevantes a los M.C.I, La emisión del ruido se produce por la contribución de diversas fuentes entre las cuales destacamos el ruido de combustión, el ruido mecánico y el aerodinámico. (Unicersitat Politecnica de Valencia, 2011)

En funcionamiento urbano el ruido del motor prevalece sobre las demás fuentes, mientras que el funcionamiento asociado al cuerpo del vehículo asociado al cuerpo del vehículo es más importante en el funcionamiento extra urbano. (Unicersitat Politecnica de Valencia, 2011) Fuentes de ruido en motores. La emisión de ruido en motores de combustión interna alternativos se produce principalmente a través de las inestabilidades producidas por la interacción de un fluido en movimiento con otro fluido inicialmente en reposo. En los motores dicha inestabilidad puede ser provocada bien por las variaciones del caudal del fluido en los extremos de los colectores de admisión y escape. (Unicersitat Politecnica de Valencia, 2011) Soluciones en el sistema de transmisión. Dado que las soluciones en la fuente son más complejas ya que se debe tomar medidas acerca del motor como a cualquier otro elemento ligado a las prestaciones del motor (colector, turbina, y sistemas de pos tratamiento de gases), además que estos elementos están destinados a la eficiencia del motor en cuanto a emisiones contaminantes y consumo resulta en pocas posibilidades de modificar la fuente. (Unicersitat Politecnica de Valencia, 2011)

\section{Silenciador.}

El ruido que se produce al chocar los gases quemados con el aire exterior se reduce merced a la interposición del silenciador, que les resta velocidad y fuerza de manera gradual durante el trayecto hasta el punto de expulsión a la atmosfera. Esto se consigue aumentando el recorrido y el espacio que van ocupando las fases por medio de divisiones o tanques perforados, que permiten su dilatación suavemente para que salgan al aire libre de modo continuo y a poca presión. (Arias Paz, 2006) 
Las disposiciones interiores de los conjuntos de escape son muy variadas, pero que ya han dejado de ser elementos secundarios construidos a capricho, puesto que se han convertido en órganos estudiados por una técnica compleja, con objeto de obtener el máximo silencio con la mínima perdida de potencia a todas las velocidades y cargas del motor. (Arias Paz, 2006)

Para caracterizar el funcionamiento de los silenciadores es habitual emplear dos magnitudes, una conocida como perdida de inserción(IL) la cual es un parámetro que se da cuenta del comportamiento acústico del silenciador definiéndose como la diferencia en decibelios entre los niveles de potencia sonora medidos en un punto antes y después de que el silenciador actué luego de una fuente de ruido dada, y el otro parámetro es la perdida de transmisión (TL) permitiendo evaluar el silenciador de manera aislada del resto del sistema, definiéndose como el cociente entre la potencia acústica incidente y la potencia acústica asociada a la onda transmitida por el mismo hacia un extremo anecoico. (Unicersitat Politecnica de Valencia, 2011)

\section{Tipos de silenciadores.}

El funcionamiento de los silenciadores varía dependiendo el tipo al que este pertenezca dentro de los cuales tenemos:

Silenciador de interferencia. Usado hace bastantes años consistía en un laberinto que amortiguaba las ondas sonoras. Se perdía bastante potencia en el motor al representar una fuerte contrapresión en el escape. No cambia la frecuencia de la onda. Baja la presión sonora. (Prieto Fernandez, Alonso Hidalgo, \& Luengo J., 2007)

Silenciador de Absorción. Es un tubo perforado rodeado de un material aislante acústico (lana de vidrio) que amortigua la onda. Para motores tiene el gran inconveniente que se deteriora con los gases de escape. (Prieto Fernandez, Alonso Hidalgo, \& Luengo J., 2007)

Silenciador de Expansión. Es una cámara vacía, donde los gases se expanden e interfieren, entre si las ondas sonoras de tal manera que se consigue una amortiguación. Para determinadas frecuencias va bastante bien, y se suele usar de forma complementaria con el resonador. (Prieto Fernandez, Alonso Hidalgo, \& Luengo J., 2007)

\section{Contrapresión en el sistema de escape.}

La diferencia entre la presión media en el tubo de escape y la presión atmosférica es la denominada contrapresión en el sistema de escape. El valor de la contrapresión es una medida de la resistencia al flujo libre de los gases a través de los diferentes componentes del sistema de escape; éste parámetro permite evaluar las restricciones ocasionadas por el silenciador, tuberías demasiado largas, tuberías de diámetro demasiado pequeño, cambios de dirección bruscos, etc. (Paucar Quinteros \& Toapanta Jaramillo, 2011) 
Valores altos en la contrapresión indican una gran resistencia al flujo de los gases de escape mientras que los valores bajos de contrapresión indican una resistencia al flujo muy baja. Generalmente los fabricantes de motores indican los valores máximos admisibles de contrapresión en el sistema de escape de manera que el funcionamiento del motor no se vea afectado. (Paucar Quinteros \& Toapanta Jaramillo, 2011)

\section{Influencia de la contra presión en la sobrealimentación.}

Los motores sobrealimentados tienen mayores problemas en su funcionamiento que los motores naturalmente aspirados cuando se presenta un aumento de la contra presión en el sistema de escape. En el turbo compresor se aprovechan los gradientes de temperatura y presión entre la entrada y la salida de la turbina para producir energía cinética que hace girar al eje que une la turbina con el compresor, este último eleva la presión del aire que es enviado hacia la admisión del motor. (Paucar Quinteros, y otros, 2011)

Mientras mayor sea la contra presión en los elementos del sistema de escape, menores serán los gradientes de presión y temperatura entre la entrada y la salida de la turbina por lo que se tendrá menor energía cinética para impulsar al compresor y por ende menor cantidad de aire será enviada al motor por unidad de tiempo. (Paucar Quinteros, y otros, 2011)

Una disminución del aire disponible para la combustión provocará una disminución en la potencia del motor, incremento en las emisiones contaminantes e incremento del consumo del combustible. (Paucar Quinteros, y otros, 2011)

Dado que el vehículo Zotye T600 posee sobrealimentación en sus dos versiones, esta consideración es aplicable al vehículo teniéndola en cuenta para seleccionar las alternativas para evitar un aumento de la contrapresión en el sistema de escape.

\section{Importancia del problema.}

Normativas nacionales buscan dar impulso a empresas del país mediante requerimientos de porcentajes mínimos permitidos de componentes de autopartes diseñados y manufacturados aquí, por tal motivo cada vez se requiere que empresas puedan estar a la altura de proveedores internacionales en los diferentes componentes automotrices.

Entre las diferentes partes que hoy en día se producen se encuentran los múltiples de escape los cuales entre sus componentes tienen a los silenciadores, estos componentes para poder ser validados por la empresa multinacional requieren contar con ciertos mínimos de calidad estos requisitos se los verifica mediante ciertos protocolos fijados por la marca los cuales metódicamente son comprobados.

Una de las principales herramientas de la ingeniería que hoy en día se utiliza es el análisis CFD el cual permite desarrollar un modelo computacional que sea capaz de remplazar a un 
modelo experimental y de este modo bajo criterios ingenieriles poder determinar el comportamiento: térmico, estructural, acústico de los diferentes componentes.

De este modo es necesario lograr encontrar un modelo computacional que permita validar y sugerir cambios a efectuarse dentro de prototipos de escapes desarrollados en el pasado, para poder optimizar tiempo y recursos.

\section{Metodología.}

Figura 1. Proceso metodológico.

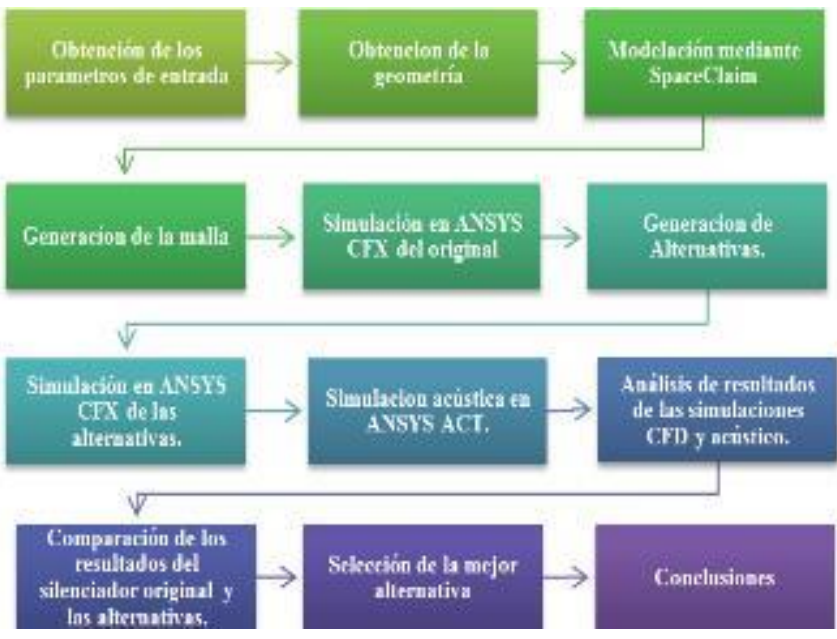

Fuente: Autores, Ecuador, 2017.

Como punto de partida, se inició con la obtención de los valores de las cargas aplicadas al sistema para su simulación CFD y acústica, para lo cual se recurrirá al uso de distintos instrumentos de medición adecuados para los parámetros de entrada al sistema en el presilenciador, los cuales son la temperatura de los gases de escape, la velocidad de flujo de los gases y la velocidad de giro del motor para poder obtener distintas mediciones en un determinado rango de rpm.

Las mediciones en distintos regímenes de funcionamiento se realizaran para poder obtener una curva que sirva de referencia, esto a fin de caracterizar el parámetro de la velocidad mediante una curva que describa su comportamiento en función de las rpm para obtener la velocidad en distintos estados de carga del motor y pode utilizar los que sean más convenientes al momento de realizar las simulaciones. 


\section{Obtención de los valores.}

Para la obtención de los valores de velocidad se utilizó de un anemómetro digital de marca Peakmeter modelo MS6252A el cual fue colocado en el sistema de escape en 3 distintos puntos debido a que la velocidad de obtención de datos es instantánea obteniendo valores a lo largo del sistema, teniendo valores para compararlos con la simulación CFD, en 2 vehículos Zotye T600 uno con una cilindrada total de 1498cc y el otro con 1997cc. En cambio para la obtención de los valores de temperatura se utilizó un multímetro digital Vici VC99 con función de medición de temperatura mediante una sonda bimetálica.

Figura 2. Anemómetro digital peakmeter modelo ms6252a y multímetro digital vici vc99
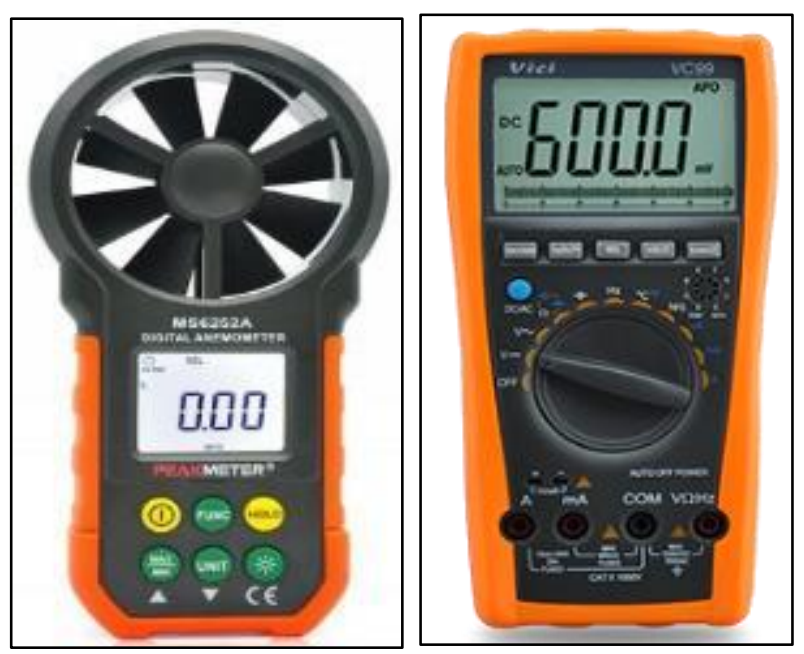

Fuente: Autores, Ecuador, 2017.

Proceso de medición. Se tomaron 5 mediciones de velocidad para cada velocidad de giro del motor en 3 puntos del sistema de escape de las cuales para realizar la simulación se utilizara la media de estas mediciones, en cuanto a las temperaturas se realizó solamente 1 medición para cada rango de velocidades debido que la medición de toma más tiempo para poder obtener el dato y el tiempo que demora la sonda del multímetro en tener una medición fija de temperatura. 
Figura 3. Grafica de regresión no lineal Zotye 1.5T

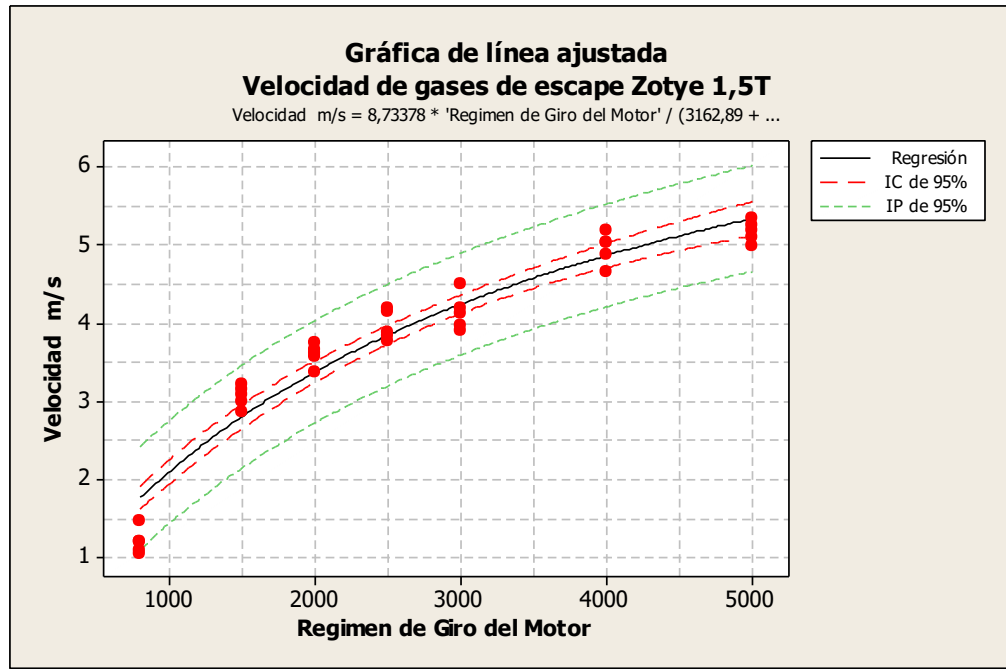

Fuente: Autores, Ecuador, 2017.

Figura 4. Grafica de regresión no lineal Zotye 2.0T

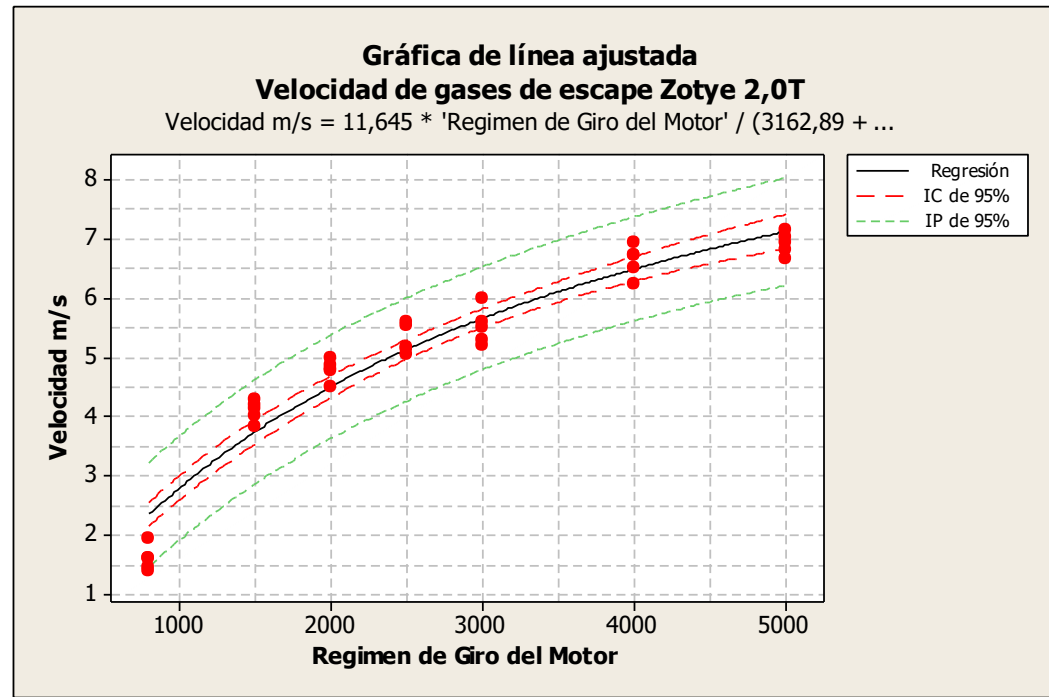

Fuente: Autores, Ecuador, 2017. 
Figura 5. Mediciones de velocidades y temperaturas en las 2 versiones del vehículo Zotye T600.

\begin{tabular}{|c|c|c|c|c|c|c|c|c|}
\hline \multirow{3}{*}{ RPM } & \multicolumn{8}{|c|}{ Versión del vehiculo } \\
\hline & \multicolumn{4}{|c|}{$1498 c c T$} & \multicolumn{4}{|c|}{$1997 \propto \mathrm{T}$} \\
\hline & Ve & $\mathrm{Vm}$ & Vsalida & $\mathbf{T}$ & Ve & $V m$ & Vsalids & $\mathbf{T}$ \\
\hline \multirow{5}{*}{800} & 1.46 & 1.96 & 1.18 & \multirow{5}{*}{170} & 1.95 & 2.61 & 1.57 & \multirow{5}{*}{19} \\
\hline & 1.08 & 1.58 & 0,95 & & 1.44 & 2,11 & 1.26 & \\
\hline & 1.19 & 1.69 & 1,01 & & 1,59 & 2,25 & 1,35 & \\
\hline & 1,05 & 1.55 & 0,93 & & 1.40 & 2.07 & 1.24 & \\
\hline & 1.20 & 1.7 & 1,02 & & 1.60 & 2.27 & 1,36 & \\
\hline \multirow{5}{*}{1500} & 3,00 & 3,5 & 2,10 & \multirow{5}{*}{204} & 4,00 & 4,67 & 2,30 & \multirow{5}{*}{233} \\
\hline & 3,15 & 3,65 & 2,19 & & 4,20 & 4,87 & 2.92 & \\
\hline & 2,87 & 3,37 & 2,02 & & 3.83 & 4,49 & 2,70 & \\
\hline & 3.09 & 3.59 & 2,15 & & 4.12 & 4.79 & 287 & \\
\hline & 3.21 & 3,71 & 2.23 & & 428 & 495 & 297 & \\
\hline \multirow{5}{*}{2000} & $3,5 ?$ & 4,03 & 2,44 & \multirow{5}{*}{245} & 4,76 & 5,43 & 3,26 & \multirow{5}{*}{$28 \%$} \\
\hline & 3,66 & 4,16 & 2,50 & & 4,88 & 5,55 & 3,33 & \\
\hline & 3,37 & 3.87 & 2,32 & & 4.49 & 5,16 & 3.10 & \\
\hline & 3,75 & 4.25 & 2,55 & & 5.00 & 5,67 & 3,40 & \\
\hline & 3,61 & 411 & 247 & & 481 & 5,48 & 3.29 & \\
\hline \multirow{5}{*}{2500} & 3,88 & 4,38 & 2,63 & \multirow{5}{*}{294} & 5,17 & 5,84 & 3,50 & \multirow{5}{*}{343} \\
\hline & 4,20 & 4,7 & 2,82 & & 5,60 & 6,27 & 3,76 & \\
\hline & 4,15 & 4,65 & 2,79 & & \begin{tabular}{|l|}
5.53 \\
\end{tabular} & 6,20 & 3,72 & \\
\hline & 3,35 & 4,35 & 2,61 & & 5,13 & 5,80 & 3,48 & \\
\hline & 3,78 & 4,28 & 2,57 & & 5,04 & 5,71 & 3,42 & \\
\hline \multirow{5}{*}{3000} & 3,91 & 4,41 & 2,65 & \multirow{5}{*}{353} & 5,21 & 5,88 & 3,53 & \multirow{5}{*}{411} \\
\hline & 3.98 & 4.48 & 2,69 & & 5,31 & 5,97 & 3.58 & \\
\hline & 4,13 & 4,63 & 2,78 & & 5,51 & 6,17 & 3,70 & \\
\hline & 4,20 & 4,7 & 2,82 & & 5,60 & 6,27 & 3,76 & \\
\hline & 4,50 & 5 & 3,00 & & 6,00 & 6,67 & 4,00 & \\
\hline \multirow{5}{*}{4000} & 467 & 5,17 & 3,10 & \multirow{5}{*}{423} & 6,23 & 6.89 & 4,14 & \multirow{5}{*}{484} \\
\hline & 5.20 & 5.7 & 3,42 & & 6.93 & 7.60 & 456 & \\
\hline & 5,04 & 5,54 & 3,32 & & \begin{tabular}{|l}
6,72 \\
\end{tabular} & 7,39 & 4.43 & \\
\hline & 5.05 & 5.55 & 3,33 & & 6.73 & 7.40 & 4.44 & \\
\hline & 4,89 & 5,39 & 3,23 & & 6,52 & 7,19 & 4,31 & \\
\hline \multirow{5}{*}{5000} & 5,20 & 5,7 & 3,42 & \multirow{5}{*}{508} & 6,93 & 7,60 & 456 & \multirow{5}{*}{582} \\
\hline & 5,00 & 5,5 & 3,30 & & 6,67 & 7,33 & 4.40 & \\
\hline & 5,27 & 5,77 & 3.46 & & \begin{tabular}{|l|}
7,03 \\
\end{tabular} & 7,69 & 4,62 & \\
\hline & 5,11 & 5,61 & 3,37 & & 6,81 & 7,48 & 4,49 & \\
\hline & 5,36 & 5,86 & 3,52 & & \begin{tabular}{|l}
7.15 \\
\end{tabular} & 7,81 & 4,69 & \\
\hline
\end{tabular}

Fuente: Autores, Ecuador, 2017.

\section{Modelación geometría externa del tubo de escape y el silenciador.}

Para poder generar la geometría en software CAD para su posterior simulación CAE se requirió contar con un ejemplar del silenciador para poder utilizar el método de ingeniería inversa con la utilización de un escáner 3D para poder digitalizar la parte exterior del silenciador y las distintas curvaturas del tubo de escape para poder reproducir con precisión las curvaturas que estas poseen sin errores.

Para esto se llevó las partes al Centro de Fomento Metalmecánico y Carrocero ubicado en la ciudad de Ambato proceso que duro 3.5h se realizó con un escáner de laser por puntos el cual genero un archivo en formato STL con la nube de puntos generados en forma de superficie obtenida por triangulación Delunay y reparándola en SpaceClaim. 
Figura 6. Tubo de ingreso al silenciador en nube de puntos del formato STL

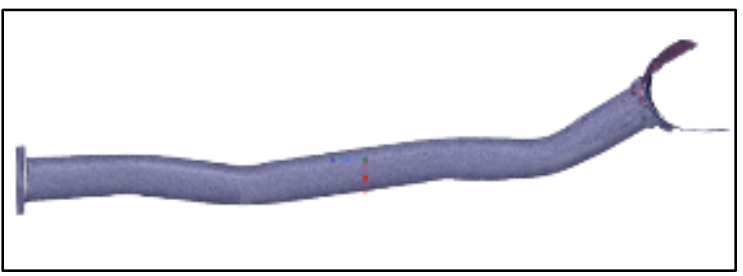

Fuente: Autores, Ecuador, 2017

Figura 7. Tubo de ingreso al silenciador en superficies generadas por la triangulación Delunay

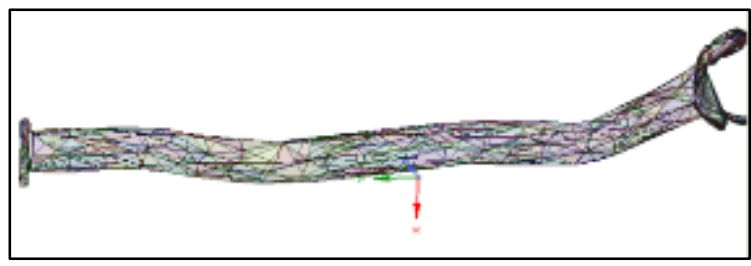

Fuente: Autores, Ecuador, 2017

\section{Modelación geometría interna del pre-silenciador y el silenciador.}

Para la geometría interna del silenciador se accedió a INDAPARTS C.L. (Industria Ecuatoriana De Partes Y Accesorios) ubicada en la ciudad de Quito provincia de Pichincha, en la cual se ensambla el pre-silenciador y silenciador del sistema de escape, los mismos que están fabricados de acero inoxidable AISI 409 en distintos espesores. (Masaquiza Moyulema \& Morales Villarroel, 2017)

Debido a que la empresa no dispone de los planos de los elementos fue necesario realizar un levantamiento de planos de los componentes del silenciador tanto individualmente como en conjunto, además se tuvo la oportunidad de observar de cerca el proceso de fabricación el cual doto una versión más clara de la estructura interna del silenciador así como las decisiones de diseño que tomaron parte en la creación del actual silenciador del Zotye T600.

Figura 8. Geometría 3D interna del silenciador acoplado a los tubos de entrada y salida.

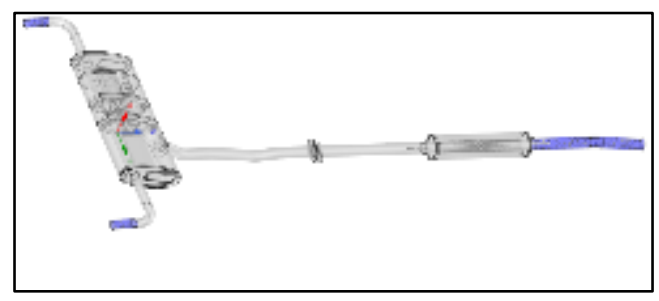

Fuente: Autores, Ecuador, 2017 


\section{Generación de la malla.}

Dado que se va a realizar una simulación con transferencia de calor se debe generar la malla tanto para el sólido del sistema de escape así como del volumen del fluido.

El número de elementos en la malla del silenciador original y las alternativas se puede observar en la figura 9.

Figura 9.Número de elementos de la malla en los diseños del silenciador.

\begin{tabular}{lc}
\hline \multicolumn{2}{l}{ Número de elementos de la malla } \\
\hline Original & 4093724 \\
Alternativa 1 & 3858936 \\
Alternativa 2 & 3871322 \\
\hline
\end{tabular}

Fuente: Autores, Ecuador, 2017

Calidad ortogonal. Con ANSYS Meshing podemos generar el mallado requerido el cual posee ciertos criterios que nos permiten evaluar la calidad de los elementos mallados en CFD para algoritmos de mallado no ensamblados, se utiliza criterios de comprobación basados en el volumen de los elementos. Para algoritmos de mallado de ensamblaje, utiliza un criterio de comprobación de forma basado en la calidad ortogonal, esta opción de preferencia recomendada para todas las simulaciones de CFD. (SAS IP.inc, 2015)

Figura 10. Calidad ortogonal del silenciador original.

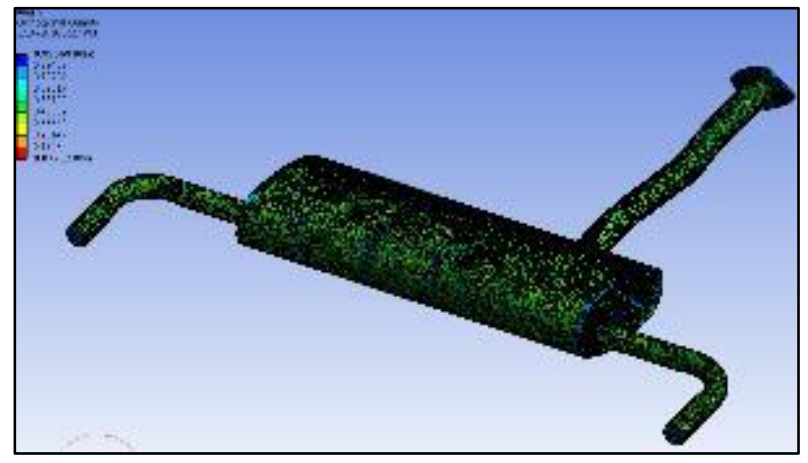

Fuente: Autores, Ecuador, 2017. 
Figura 11. Histograma de calidad ortogonal de los elementos de la malla.

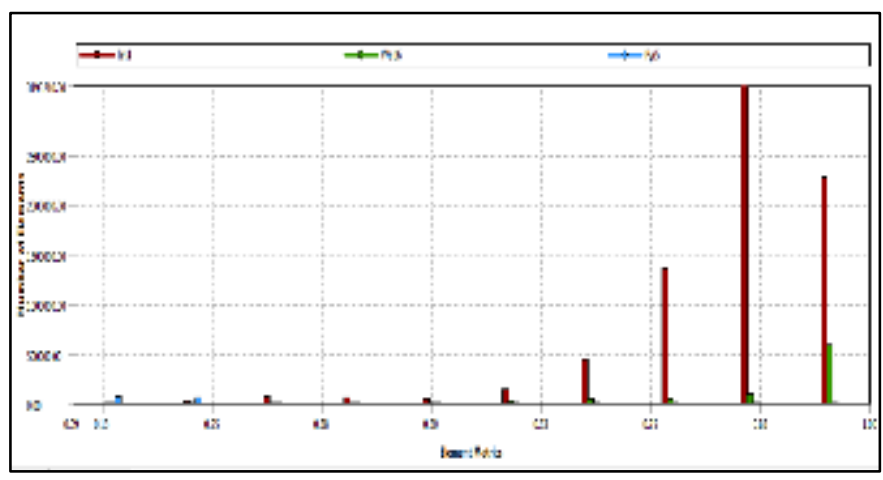

Fuente: Autores, Ecuador, 2017.

\section{Proceso de Simulación.}

Debido al empleo de transferencia de calor en la simulación del modelo CFD el gasto computacional se incrementa considerablemente por lo cual se utilizó un Workstation portátil de Lenovo, el modelo Thinkpad P50 con procesador Intel Xeon E3 1505v5 2.7Ghz, tarjeta de video Nvidia Quadro M2000M 4Gb y 16GB de memoria RAM.

Se ha considerado realizar 3 simulaciones para probar el sistema de escape en 3 regímenes de giro del motor distintos tomándose un valor en ralentí (800rpm), carga media (3000rpm) y carga completa (5000rpm) para cada uno de los modelos de vehículos ya que al variar el cilindraje también varían los parámetros a tener en cuenta para la simulación CFD que va a ser en función del tiempo para poder constatar las características de funcionamiento del silenciador en cuanto a la transferencia de calor de los gases de escape al silenciador en un periodo de tiempo.

\section{Simulación en ANSYS CFX.}

Debido a que será necesario comparar los resultados de distintas simulaciones se realizó todas en el mismo entorno de trabajo de ANSYS en un archivo común, a partir de lo cual se tendrá la geometría, la simulación en CFX y la simulación en ANSYS ACT independientes entre sí pero con relación entre ellas.

Se debe cargar la geometría, y exportarla hacia el módulo CFX para el cálculo CFD y al finalizar visualizar los resultados en un mismo modulo para comparar entre si las distintas geometrías y parámetros.

Se seleccionaron las características de la simulación, en este caso es de tipo "Transitorio" por lo cual la simulación se desarrollara en un intervalo de tiempo, y definiendo el intervalo en el 
que transcurre y el tamaño de las divisiones que se le realizaran durante la simulación a utilizar.

Se selecciona el modelo de transferencia de calor a utilizarse, el modelo elegido es "Thermal Energy" debido a que es la selección por defecto en modelos de baja velocidad inferiores a 0.3Match debido a que en estos la compresibilidad de los gases no es un factor determinante. (Sharcnet, 2015)

Se selecciona el dominio correspondiente al solido del silenciador y es definido como acero inoxidable 409 correspondiente al material del cual está fabricado el silenciador debido a que proporciona una baja transferencia de calor, mayor resistencia a la oxidación y corrosión. Para el análisis de transferencia de calor se toma en cuenta las propiedades físicas del material específicamente las térmicas debido a que en base a estas el programa calcula la difusividad térmica que nos indica que tan rápido un material responde a los cambios térmicos del ambiente, ya que es la capacidad del material de transportar energía a través de la conducción térmica en relación a su capacidad para almacenarla. (CAE Associates Inc., 2016)

\section{Simulación en ANSYS ACT.}

Para la simulación acústica se utilizara un módulo acústico generado para ANSYS ACT usando como base el ANSYS Mechanical con una simulación de respuesta armónica de vibraciones, debido a la extensión acústica se generara una barra de herramientas adicional en la interfaz de ANSYS permitiendo definir las condiciones de frontera y parámetros acústicos.

En ajustes del análisis se debe cambiar el tipo de espaciamiento de la frecuencia a logarítmico debido a que la intensidad de sonido en decibeles se basa en una escala logarítmica. El rango de frecuencia se debe establecer entre 0 y $3000 \mathrm{~Hz}$ debido a que es el rango de frecuencias utilizado mayoritariamente en los trabajos en silenciadores utilizados en vehículos.

Debe definirse el número de intervalos de solución lo cual influye directamente en el tiempo de solución para esta simulación se utilizará 20 intervalos para poder generar la gráfica de la perdida de transmisión del sonido en función de la frecuencia. También se debe cambiar el método de solución al completo debido a que es el que nos ofrece resultados más precisos.

Dado que las condiciones de absorcion de las ondas permiten modelar una pequeña porcion del dominio del fluido y asumir que las ondas propagadas son emitidas fuera del sistema y no reflejadas de nuevo al interior ya que se consideraria al silenciador como cerrado, Para definir al silenciador como abierto en sus extremos debemos hacer uso de condiciones de radiacion para absorber las ondas salientes del sistema. (ANSYS, 2016)

\section{Generación de alternativas.}

De acuerdo con los resultados de trayectorias en streamlines en la simulación en la figura 12 se puede concluir que existe una subutilización de las cámaras intermedias, mientras que la mayoría del flujo se concentra en la primera y segunda cámara, con lo cual en esta zona se 
producen puntos en los que se genera una transferencia de calor más rápida y fácilmente apreciable.

Figura 12. Comparación de velocidades del fluido mediante streamlines, diseño original (izquierda), alternativa 1(centro) y la alternativa 2 (derecha).

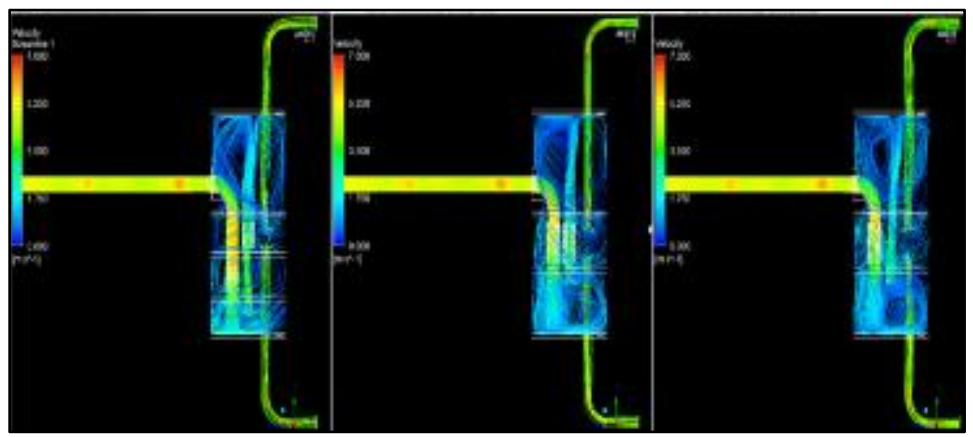

Fuente: Autores, Ecuador, 2017.

Debido a estas consideraciones de la simulación se optó por la generación de 2 modelos de silenciador alternativos para ser probados mediante simulación CFD bajo las mismas condiciones que el modelo original, la cual servirá de punto de partida para probar el comportamiento de estos en comparación con el original, buscando principalmente una distribución más uniforme de la temperatura en las cámaras del silenciador y una circulación más óptima del silenciador para que el flujo de gases no se concentren sobre las primeras cámaras.

Figura 13. Alternativa número 1 del silenciador.

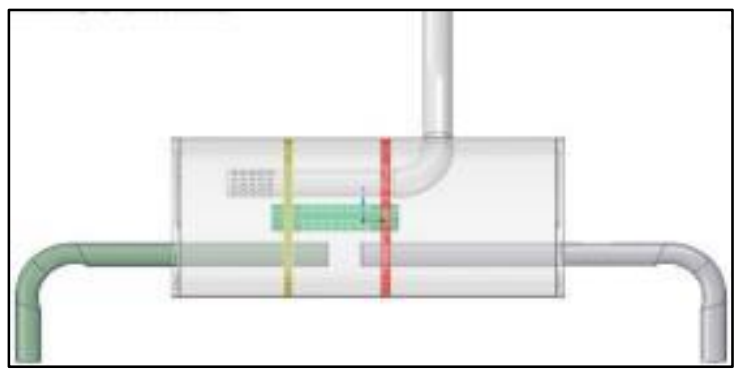

Fuente: Autores, Ecuador, 2017. 
La primera alternativa se basa en 2 efectos de las distintas configuraciones de silenciadores. La primera de estas es la longitud de las cámaras con lo que destaca la influencia de esta en la atenuación de ruido, al aumentar la longitud el pico de atenuación se mantiene pero la frecuencia con la que aparece este pico de atenuación se reduce. (Davis, Strokes, Moore, \& Stevens, 1954)

Figura 14. Efecto de la longitud de la cámara sobre la frecuencia de atenuación máxima.

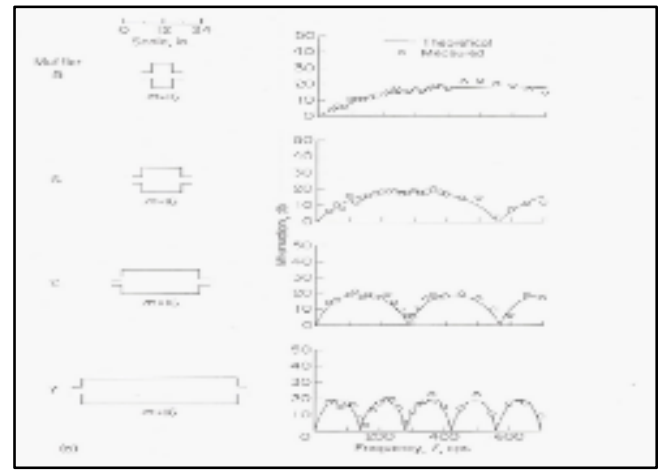

Fuente: Autores, Ecuador, 2017.

La segunda consideración es el efecto del número de cámaras, el cual nos indica que al aumentar estas se produce un aumento en la atenuación máxima, sin embargo, al darse solo un pequeño aumento entre de 2 a 3 cámaras se determina que un número mayor de cámaras no logra generar un aumento significativo de la atenuación. (Davis, Strokes, Moore, \& Stevens, 1954)

Figura 15. Efecto del número de cámaras sobre la atenuación máxima generada.

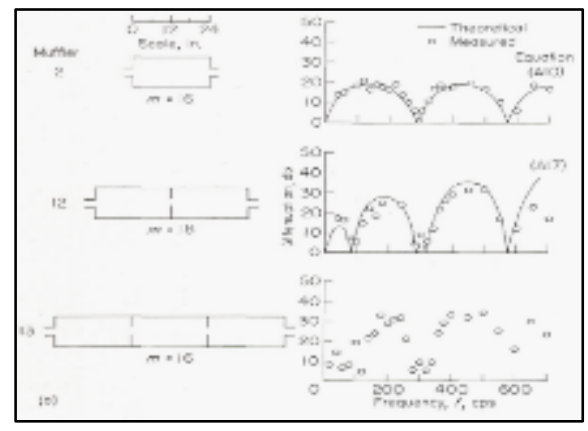

Fuente: Autores, Ecuador, 2017.

En la generación de la segunda alternativa del silenciador se tomó en cuenta los mismos efectos considerado es en la primera alternativa del número de cámaras sobre la atenuación 
máxima generada y efecto de la longitud de la cámara sobre la frecuencia de atenuación, además aumentar el flujo del fluido hacia la última cámara del silenciador eliminando las perforaciones en tubo que conecta la primera y la tercera cámara. (Davis, Strokes, Moore, \& Stevens, 1954)

Figura16. Alternativa número 2 del silenciador.

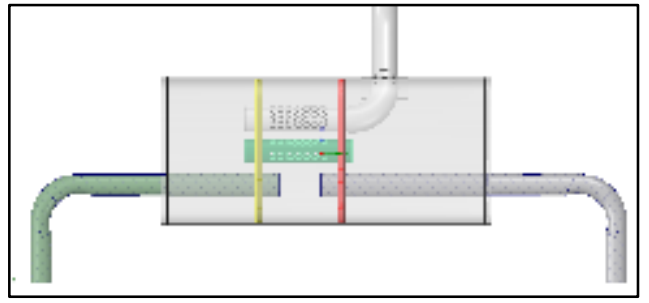

Fuente: Autores, Ecuador, 2017.

\section{Resultados.}

\section{Análisis del silenciador original y las alternativas propuestas.}

Figura 17. Imagen termográfica del comportamiento de la distribución de calor en la superficie del silenciador del Zotye T600.

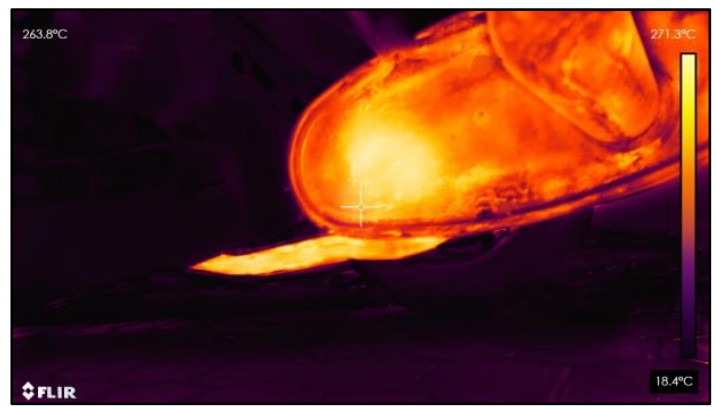

Fuente: Autores, Ecuador, 2017.

Figura 18. Comparación de velocidades a la salida derecha del silenciador mediante histograma, diseño original (azul) y la alternativa 1 (rojo).

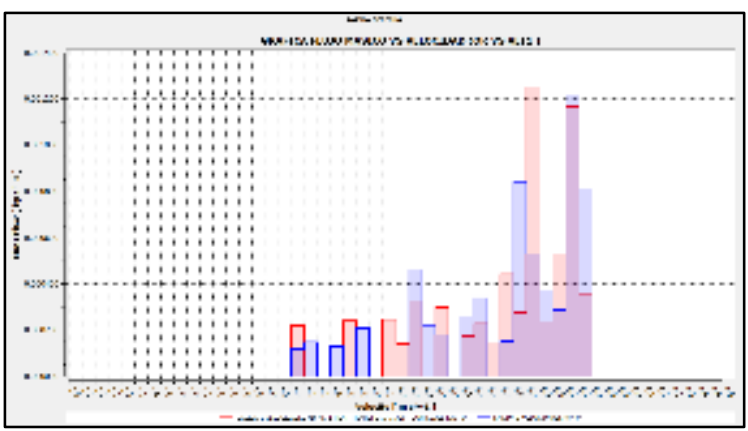

Fuente: Autores, Ecuador, 2017. 
Figura 19. Comparación de velocidades a la salida izquierda del silenciador mediante histograma, diseño original (azul) y la alternativa 1 (rojo).

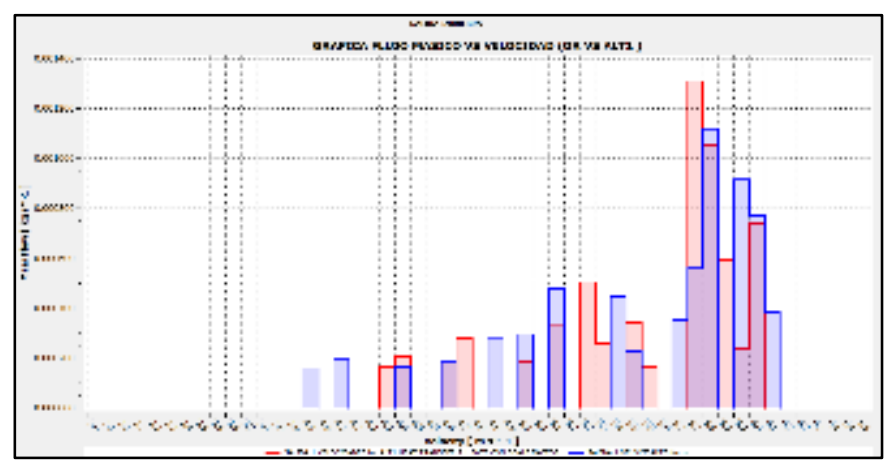

Fuente: Autores, Ecuador, 2017.

En los histogramas de comparación de las velocidades de salida entre el diseño original y la segunda alternativa en la figura 15 las velocidades en la salida derecha es ligeramente mayor en la alternativa con respecto al diseño original y del histograma de la salida izquierda de la figura 16 tiene una velocidad de salida mayor en el diseño original en comparación a la segunda alternativa.

Figura 20. Comparación de velocidades a la salida derecha del silenciador mediante histograma, diseño original (azul) y la alternativa 2 (verde).

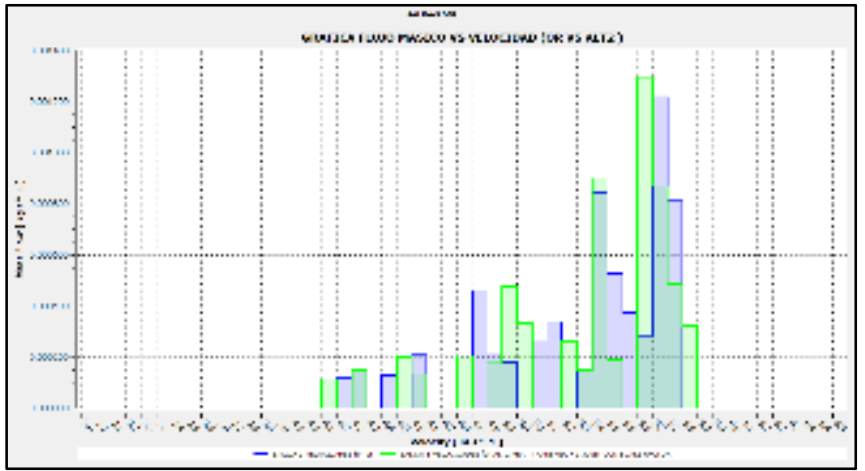

Fuente: Autores, Ecuador, 2017. 
Figura 21. Comparación de velocidades a la salida izquierda del silenciador mediante histograma, diseño original (azul) y la alternativa 2 (verde)

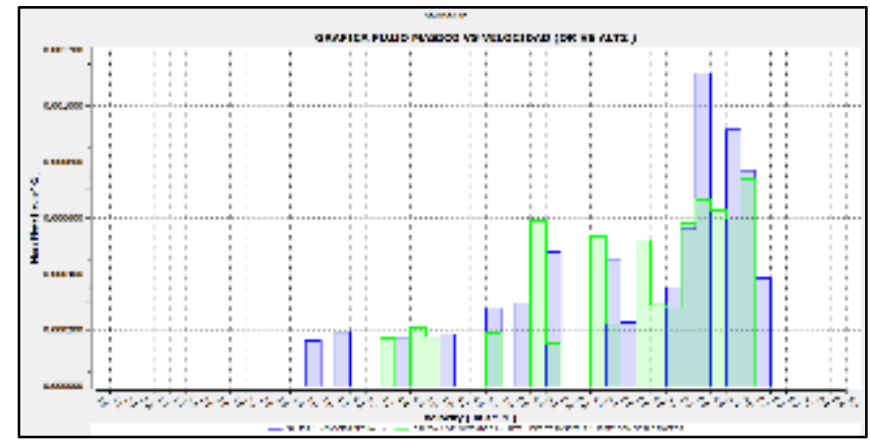

Fuente: Autores, Ecuador, 2017

De las velocidades obtenidas en los histogramas concluimos que la velocidad de salida de los gases de escape en el diseño original y las alternativas tiene variaciones de velocidades ligeras, con lo que las alternativas no presentan un cambio significativo en el funcionamiento del silenciador a pesar de la reducción del material empleado.

En la figura 17 se puede apreciar las velocidades medias a las salidas del silenciador original y las alternativas, puesto que las diferencias porcentuales entre las medias del silenciador original y las alternativas no son significativas estadísticamente se puede concluir que las alternativas no generan un freno a la salida de los gases de escape.

Figura 22. Salida derecha del silenciador.

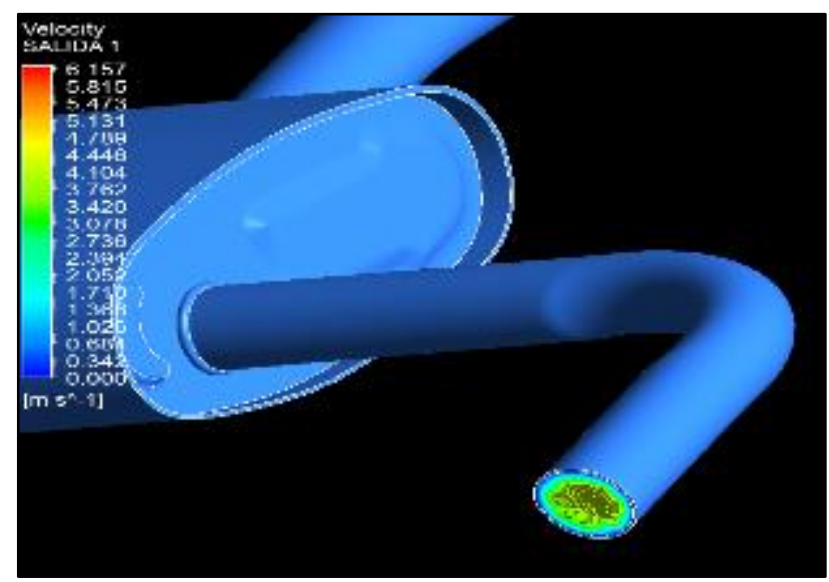

Fuente: Autores, Ecuador, 2017 
Figura 23. Comparación de los valores de velocidad en la entrada y las salidas de los silenciadores.

\begin{tabular}{|c|c|c|c|c|c|c|}
\hline \multicolumn{7}{|c|}{ VELOCIDADES $(\mathrm{m} / \mathrm{s})$} \\
\hline \multirow{2}{*}{ Eutradas } & \multirow{2}{*}{ Salidas } & Origieal & \multicolumn{2}{|c|}{ Alternativa 1} & \multicolumn{2}{|c|}{ Alternativa 2} \\
\hline & & Media & Media & Dif Origimal & Media & Dif. Origeal \\
\hline \multirow{2}{*}{$\begin{array}{l}1,7 \mathrm{~mm}- \\
800 \mathrm{rgm}\end{array}$} & Izquictds & 1,43767 & 1,42497 & $0,55 \%$ & 1,41676 & $1,45 \%$ \\
\hline & Derecha & 1,35947 & 1,36885 & $-0,69 \%$ & 1,37455 & $-1,11 \%$ \\
\hline \multirow{2}{*}{$\begin{array}{l}, 2 \mathrm{mg}- \\
3500 \mathrm{rg}=\end{array}$} & laģuerda & 4,46519 & 4,38424 & $1,81 \%$ & 4,40548 & $1,38 \%$ \\
\hline & Deracha & 4,2262 & 4,24434 & $-0,43 \%$ & 4,25323 & $.0,64 \%$ \\
\hline \multirow{2}{*}{\begin{tabular}{|c|}
$7,1 \mathrm{~m} / \mathrm{s}-$ \\
$5000 \mathrm{ryen}$
\end{tabular}} & Isquierda & 6,09894 & 6,03435 & $1,05 \%$ & 6,037 & $1,02 \%$ \\
\hline & Derecha & 5,79896 & 5,77559 & $0,40 \%$ & 5,81259 & $0,24 \%$ \\
\hline
\end{tabular}

Fuente: Autores, Ecuador, 2017.

\section{Comparación de temperaturas en un punto.}

Para determinar la variación entre las temperaturas de los diseños y en las distintas velocidades. Se utilizó la herramienta "Probe" en la coordenada $(0.0559597,0.33703 ; 0.102028)$ en los modelos CAD y se obtuvo los valores de la tabla 3 en la cual se indica la diferencia porcentual entre la temperatura medida en el punto en el silenciador original y las alternativas planteadas de las cuales la segunda alternativa provee una mayor reducción de la temperatura transferida al solido en ese punto.

Figura 24. Coordenada de prueba en el silenciador para medición de temperatura.

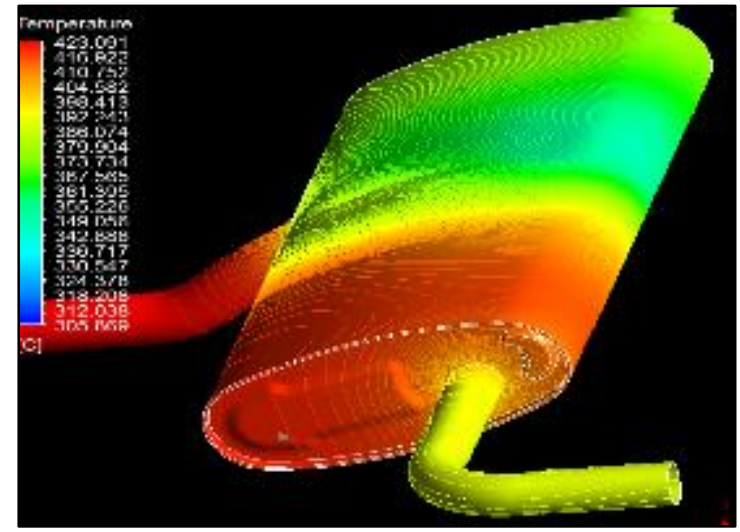

Fuente: Autores, Ecuador, 2017.

Figura 25. Comparación de los valores temperatura transferida al sólido en un punto de la tapa lateral silenciador original y las alternativas.

\begin{tabular}{|c|c|c|c|c|}
\hline \multicolumn{5}{|c|}{ Iemperatura en el punto de prueba } \\
\hline & & $\begin{array}{l}1,7 \mathrm{~m} / \mathrm{s}- \\
800 \mathrm{rpm}\end{array}$ & $\begin{array}{l}5,2 \mathrm{~m} / \mathrm{s}- \\
3500 \mathrm{rpm}\end{array}$ & $\begin{array}{l}7,1 \mathrm{~m} / \mathrm{s}- \\
5000 \mathrm{rpem}\end{array}$ \\
\hline Original & Tecuperatura ${ }^{\circ} \mathrm{C}$ & 418,364 & 421,312 & 421,321 \\
\hline \multirow{2}{*}{ Alternativa 1} & Tecruperatura ${ }^{\circ} \mathrm{C}$ & 415,974 & 419,804 & 420,429 \\
\hline & Difereacia Original & $0,57 \%$ & $0,36 \%$ & $0,21 \%$ \\
\hline \multirow{2}{*}{ Alternativa 2} & Tecrpesatura ${ }^{\circ} \mathrm{C}$ & 395,354 & 406,386 & 408,162 \\
\hline & Difereacia Original & 5,5036 & $3,54 \%$ & 3,1236 \\
\hline
\end{tabular}


Fuente: Autores, Ecuador, 2017.

\section{Comparación de presiones en un punto.}

En la figura 19 se observa que las presiones absolutas entre los diseños se mantienen en valores similares con ligeras variaciones como consecuencia de esto se puede observar que al reducir las cámaras de 4 a 3 no se da un aumento de las presiones internas en las cámaras del silenciador.

Figura 26. Plano de selección de presión interna para comparación.

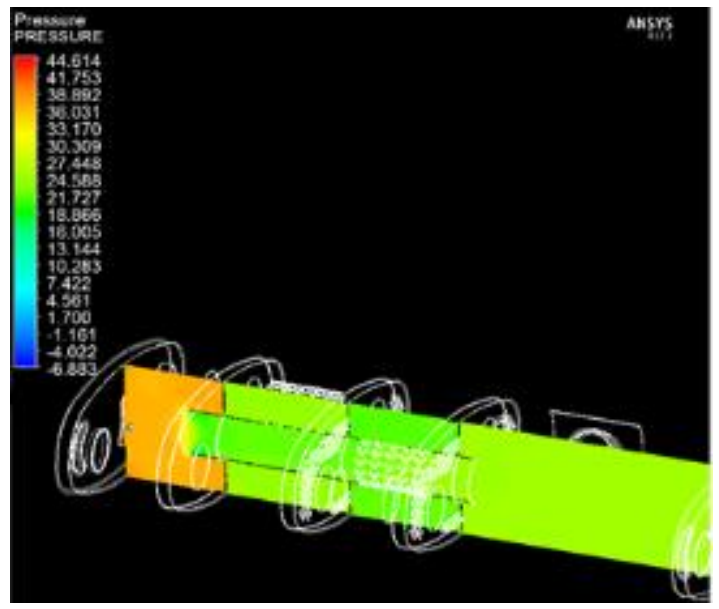

Fuente: Autores, Ecuador, 2017

Figura 27. Comparación de los valores de pérdida de transmisión en función de la frecuencia para el silenciador original y las alternativas.

\begin{tabular}{|c|c|c|c|}
\hline \multicolumn{4}{|c|}{ Presiones (Pa) } \\
\hline ENTRADAS & $800 \mathrm{rpm}$ & $3500 \mathrm{rpm}$ & $5000 \mathrm{rpm}$ \\
\hline Original & 101329,1585 & 101367,2836 & 101403,078 \\
\hline Alternativa 1 & 101328,5674 & 101359,563 & 101389,8756 \\
\hline & $0,001 \%$ & $0,008 \%$ & $0,013 \%$ \\
\hline Alternativa 2 & 101328,6931 & 101360,395 & 101389,9854 \\
\hline & $0,000 \%$ & $0,007 \%$ & $0,013 \%$ \\
\hline
\end{tabular}

Fuente: Autores, Ecuador, 2017. 


\section{Comparación del flujo másico en las entradas y salidas en el silenciador.}

En la figura 20 se puede observar la comprobación de la cantidad de flujo en la entrada y la cantidad total obtenida en las salidas para determinar que se cumple con la ley de continuidad teniendo la misma cantidad de fluido a la entrada y a la salida.

Figura 28. Comparación de los valores de pérdida de transmisión en función de la frecuencia para el silenciador original y las alternativas.

\begin{tabular}{|c|c|c|c|c|}
\hline \multicolumn{5}{|c|}{ FLUJO Mísico $(\mathrm{g} / \mathrm{s})$} \\
\hline Intradas & Salidas & Original & Alternativa 1 & Alternativa 2 \\
\hline \multirow{3}{*}{$4.04889 \mathrm{~g} s .800 \mathrm{rpm}$} & Isquierds & 2,06812 & 2,05035 & 2,0515 \\
\hline & Derecha & 1,93076 & 1,99854 & 1,99739 \\
\hline & Total & 4,04898 & 4,04889 & 4,04589 \\
\hline \multirow{3}{*}{$12.3848 \mathrm{~g}$ s. $3500 \mathrm{ppm}$} & Isquierda & 6,33483 & 6,2782 & 6,2855 \\
\hline & Derecha & 6.04994 & 6,10669 & 6,0993 \\
\hline & Total & 1238477 & 1235489 & 123548 \\
\hline \multirow{3}{*}{$16.9101 \mathrm{~g} / \mathrm{k}-5000 \mathrm{~mm}$} & Ixquierda & 8,63146 & 8,62201 & $\$, 59958$ \\
\hline & Derecha & 82786 & 8.25807 & 8,31055 \\
\hline & Total & 16,91006 & 1691008 & 16,91013 \\
\hline
\end{tabular}

Fuente: Autores, Ecuador, 2017.

Figura 29. Comparación de los valores de pérdida de transmisión en función de la frecuencia para el silenciador original y las alternativas.

\begin{tabular}{|c|c|c|c|c|c|}
\hline \multirow[b]{2}{*}{ Frocuencia } & \multirow{2}{*}{$\begin{array}{l}\text { Orizinal } \\
\text { TL(dB) }\end{array}$} & \multicolumn{2}{|c|}{ Alternativa 1} & \multicolumn{2}{|c|}{ Alternativa2 } \\
\hline & & TL(dB) & Dif. Origimal & TL(dB) & Dif. Origimal \\
\hline 10,60 & 4,31 & 4,33 & $0,32 \%$ & 4,35 & $0,91 \%$ \\
\hline 15,02 & 5,74 & 5,84 & $1,86 \%$ & 5,88 & $2,51 \%$ \\
\hline 22,59 & 6,48 & 6,59 & $1,63 \%$ & 6,65 & 2,$622 ;$ \\
\hline 33,95 & 12,17 & 11,33 & $-5,9396$ & 11,50 & $-5,56 \%$ \\
\hline$\$ 1,02$ & 22,23 & 21,98 & $-1,15 \%$ & 22,31 & $0,33 \%$ \\
\hline 75,68 & 27,17 & $28, \$ 2$ & 6,079 & 29,74 & $9,46 \%$ \\
\hline 115,25 & 27,51 & 21,00 & $-23,65 \%$ & 53,10 & $93.05 \%$ \\
\hline 173,21 & 39,84 & 35,10 & $-11,902 / 4$ & 35,60 & $-10,66 \%$ \\
\hline 260,31 & 14,70 & 39,04 & $165,51 \%$ & 34,12 & $132,02 \%$ \\
\hline 391,23 & 15,23 & 11,89 & $-21,93 \%$ & 14,27 & $-6,28 \%$ \\
\hline 587.90 & 38,68 & 19,09 & $-50,63 \%$ & 26,74 & $-30,86 \%$ \\
\hline \multicolumn{2}{|c|}{ Sfres del gritico 58} & 75,87 & 91,709 & 50,23 & $26,91 \%$ \\
\hline 1328,15 & 42,39 & 20,12 & $-52,74 \%$ & 18,02 & $-57,68 \%$ \\
\hline 1996,11 & 29,50 & 37,50 & $27,11 \%$ & 34,76 & $17,81 \%$ \\
\hline 3050,00 & 12,61 & 17,46 & 38,549 & 18,30 & $45,14 \% \mathrm{i}$ \\
\hline
\end{tabular}

Fuente: Autores, Ecuador, 2017. 
Figura 30. Gráfica de pérdida de transmisión en función de la frecuencia del presilenciador

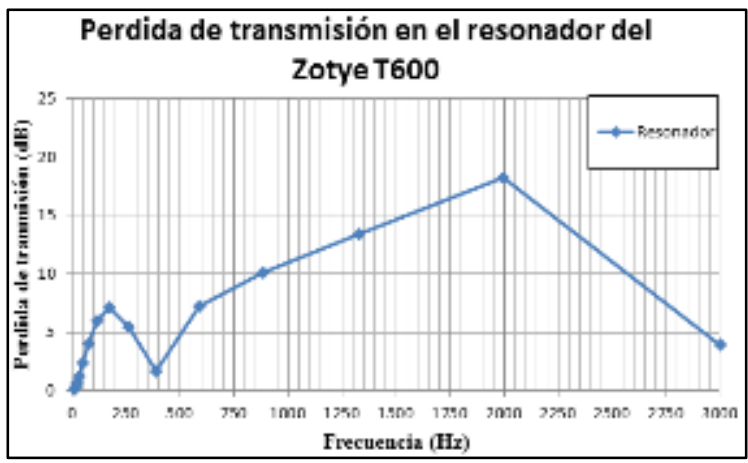

Fuente: Autores, Ecuador, 2017

Figura 31. Gráfica de pérdida de transmisión en función de la frecuencia.

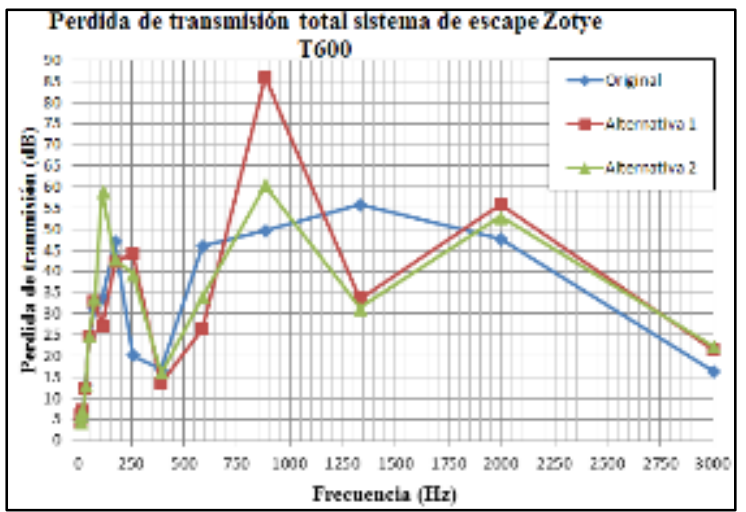

Fuente: Autores, Ecuador, 2017

\section{Discusión.}

De acuerdo a los valores obtenidos de las simulaciones se puede observar que el silenciador original presenta un comportamiento similar a las alternativas en cuanto a las presiones internas de las cámaras y las velocidades de salida del silenciador, por el contrario, en la transferencia de calor al sólido, las temperaturas internas del fluido y la atenuación de ruido.

Se puede apreciar que las alternativas poseen mejores prestaciones en especial la alternativa 2 que reduce en las zonas de mayor acumulamiento de calor hasta el 5.5\% en relación al silenciador original con un ahorro del $5.43 \%$ de material. 


\section{Conclusiones.}

- Se obtuvo información acerca del funcionamiento y utilidad de los silenciadores y la influencia de su configuración en su funcionamiento.

- Se desarrolló del modelo CAD mediante levantamiento de planos y escaneo 3D con el software SpaceClaim, generando alternativas de diseño al realizar una primera simulación se logró identificar las zonas a enfocarse para las alternativas.

- Se logró una reducción de hasta el 5.5\% de temperatura en las zonas de mayor transferencia de calor al solido en el silenciador.

- Se generó alternativas de diseño mediante el uso de elementos finitos evitando pruebas experimentales de alto costo.

- Se logró una reducción del 5.75\% y 5.43\% de material respecto al original.

- Se logró caracterizar el sistema de escape del vehículo Zotye T600 facilitando su futura manufactura.

\section{Referencias bibliográficas.}

ANSYS. (2016). CADFamily. Retrieved $12 \quad 28$, 2018, from http://www.cadfamily.com/download-pdf/ANSYS_Rotordynamics/AACTx_R160_L01_\%20Introduction\%20to\%20Acoustics.pdf

ANSYS. (2017). Ansys. Retrieved 02 24, 2018, from Simulation and Analysis: https://www.ansys.com/es-es/products/3d-design/ansys-spaceclaim/simulation-and$\underline{\text { analysis }}$

ANT. Presidencia de la República del Ecuador. (2012). Retrieved 11 17, 2017, from https://www.ant.gob.ec/index.php/programas/normas-y-reglamentos-inen/emisionescontaminantes-y-ruido/file/158-libro-6-anexo5.

CAE Associates Inc. (2016). https://caeai.com. Retrieved 03 03, 2018, from CAE Associates: https://caeai.com/blog/calculating-solution-settings-transient-thermal-analysis-part-1

Changzhou Dongfeng Agricultural Machinery Group Co., Ltd. (n.d.). DFAM. Retrieved 02 12, 2017, from DFAM: http://dftractor.es/2b-DF-15L-walking-tractor.html

Comparison of effects on technical variances of computational fluid dynamics (CFD) software based on finite element and finite volume methods. International Journal of Mechanical Sciences Jeong, W., \& Seong, J. (2014)., 78, 19-26. 
cfdninja. Ponce Segovia, I. (2017). Retrieved 12 25, 2017, from https://cfdninja.com/cfd/ansys-history/

Congress on Project Management and Engineering.Parras, D., Romero, L., Cavas, F., Nieto, J., Cañavate, F., \& FernándezPacheco. (2016).

Digitalización de la nube de puntos 3D generada por el scanner picza LPX-60 para el modelamiento y manufactura de productos industriales mediante algoritmo básico de MATLAB y software libre MESHLAB.: Cajas Naranjo, H. G. (2016). http://repositorio.espe.edu.ec/handle/21000/12056DSpace. Retrieved 02 17, 2018, from http://repositorio.espe.edu.ec/handle/21000/12056

DSpace ESPOCH Masaquiza Moyulema, W. I., \& Morales Villarroel, L. A. (2017, 08 15).. (Escuela Superior Politécnica de Chimborazo) Retrieved 03 01, 2018, from http://dspace.espoch.edu.ec/handle/123456789/7698

Fundamentos de máquinas térmicas. Oviedo: Universidad de Oviedo. Prieto Fernandez, I., Alonso Hidalgo, M., \& Luengo J., C. (2007).

Garrett. (2018). www.turbobygarrett.com. Retrieved 03 08, 2018, from https://www.turbobygarrett.com/turbobygarrett/sites/default/files/catalog/TBGCatalog-Vol-7.pdf

Ingenieria del automovil: sistemas y comportamiento dinamico Luque P, A. D. (2008).. Madrid: Paraninfo.

Machine Design. Sjodin, B. (2016, 04 18). (COMSOL, Producer) Retrieved 02 22, 2018, from http://www.machinedesign.com/fea-and-simulation/what-s-difference-between-femfdm-and-fvm

Manual práctico del automóvil (MMVIII ed.). Hermógenes Gil Martínez. (2008). Madrid: Cultural S.A.

Manual de automóviles Arias Paz, M. (2006).. Madrid, España: CIE Inversiones Editoriales Dossat 2000

Ministerio de Comercio Exterior. (2017). http://www.proecuador.gob.ec. Retrieved 12 01, 2017, from http://www.proecuador.gob.ec/compradores/oferta-exportable/automotriz/

Modeling Software Dor Engineering SpaceClaim Corporation. (2015). 3D. Retrieved 02 25, 2018, from Spaceclaim: http://www.spaceclaim.com/en/default.aspx

Motores de combustión interna alternativos.Unicersitat Politecnica de Valencia. (2011). (F. Payri González, \& J. Desantes Fernández, Eds.) Valencia, España: Editorial Reverté. 
PROECUADOR. (2017). https://www.proecuador.gob.ec. (Ministerio de comercio exterior) Retrieved $12 \quad 01, \quad 2017$, from https://www.proecuador.gob.ec/wpcontent/uploads/2017/02/PERFIL-AUTOMOTRIZ-FINAL.pdf

Rediseño del ducto de escape de los motores ALCO en las cinco estaciones de bombeo del sote Paucar Quinteros, W. D., \& Toapanta Jaramillo, A. S. (2011, 09 06). Bibdigital.epn.edu.ec. (EPN) Retrieved 12 01, 2017, from: http://bibdigital.epn.edu.ec/handle/15000/4073

Retrieved 01 08, 2018, from Sistema de Bibliotecas y Biblioteca Central: http://sisbib.unmsm.edu.pe/bibvirtualdata/publicaciones/indata/vol9_n1/a02.pdf

SAE. (2014). Sae. Retrieved 12 21, 2017, from Optimizing commercial vehiclemuffler sizevia CFD: http://articles.sae.org/13606/

SAS IP.inc. (2015). SHARCNET. Retrieved 02 03, 2018, from https://www.sharcnet.ca/Software/Ansys/17.0/enus/help/wb_msh/ds_Shape_Checking.html

Sharcnet. (2015). www.sharcnet.ca, Heat transfer. Retrieved 03 03, 2018, from https://www.sharcnet.ca/Software/Ansys/16.2.3/en-us/help/cfx_mod/i1298521.html

SIEMENS. (2017). Retrieved 01 08, 2018, from Siemens PLM Software: https://www.plm.automation.siemens.com/global/es/our-story/glossary/computeraided-design-cad/12507

SimuTechGroup. (2014). SimuTechGroup. Retrieved 03 02, 2018, from https://www.simutechgroup.com/tips-and-tricks/fea-articles/239-fea-tips-trickselement-plots-ansys-16

SpaceClaim Corporation. (2013). Spaceclaim. Retrieved 02 24, 2018, from Software For Engeneering

Spaceclaim: http://www.spaceclaim.com/en/Products/ANSYSSpaceClaim.aspx

Toyocosta. (2014, 05 20). Toyocosta. Retrieved 12 01, 2017, from http://www.toyocosta.com/blog/wp-content/uploads/2014/05/Sistema-de-escape.jpg

Technical Reports Server. Davis, D. D., Strokes, G. M., Moore, D., \& Stevens, G. J. (1954). NASA (National Advisory Committee for Aeronautics) Retrieved 09 2017, 16, from https://ntrs.nasa.gov:

https://ntrs.nasa.gov/archive/nasa/casi.ntrs.nasa.gov/19930092208.pdf

UNIVERSIDAD NACIONAL MAYOR DE SAN MARCOS Rojas Lazo, O., \& Rojas Rojas, L. (2006). 
USE OF 3D SCANNING AND REVERSE ENGINEERING FOR THE PROTOTYPING. 20th International

Universidad Rafael Urdaneta. (2011). Uru.edu. Retrieved 01 08, 2018, from http://www.uru.edu/fondoeditorial/libros/pdf/elementosfinitos/CAP\%201\%20COMPL ETO.pdf

Universidad Nacional de La Plata. Retrieved 03 01, 2018 Barreiro, M., Chilano, A., Sabelli, $\begin{array}{llll}\text { P., } \quad \text { M } & \text { Marcos, } & \text { (2012)., from }\end{array}$ https://www.ing.unlp.edu.ar/catedras/M0639/descargar.php?secc=0\&id=M0639\&id_i $\underline{\mathrm{nc}=2900}$

Vehicular Engine Design (Segunda ed.). Hoag, K., \& Dondlinger, B. (2016). Vienna: Springer Vienna.

Why MultiBody Dynamics RecurDyn. (2014).. Retrieved 01 08, 2018, from Web.archive.org: https://web.archive.org/web/20141021080603/http://www.functionbay.de/whymultibody-dynamics-simulation.html 


\section{Para citar el artículo indexado.}

Montúfar P., Moreno R., Choto L., Buenaño L. \& Escobar M. . (2018). Análisis del comportamiento acústico y optimización del material del escape de automóviles livianos equipados con turbo compresor mediante herramientas de dinámica de fluidos computacionales. Revista electrónica Ciencia Digital 2(3), 440-566. Recuperado desde: http://cienciadigital.org/revistacienciadigital2/index.php/CienciaDigital/article/view/175/15 $\underline{5}$

\section{LCiencia}

El artículo que se publica es de exclusiva responsabilidad de los autores y no necesariamente reflejan el pensamiento de la Revista Ciencia Digital.

El articulo queda en propiedad de la revista y, por tanto, su publicación parcial y/o total en otro medio tiene que ser autorizado por el director de la Revista Ciencia Digital.
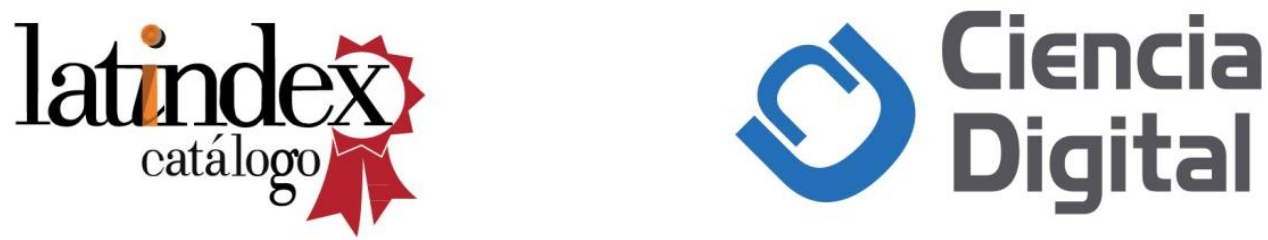